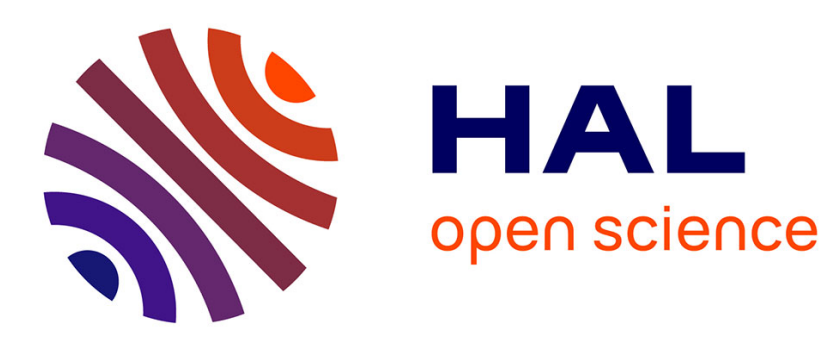

\title{
A Matched-Pulse Approach for Soft-Fault Detection in Complex Wire Networks
}

\author{
Layane Abboud, Andrea Cozza, Lionel Pichon
}

\section{To cite this version:}

Layane Abboud, Andrea Cozza, Lionel Pichon. A Matched-Pulse Approach for Soft-Fault Detection in Complex Wire Networks. IEEE Transactions on Instrumentation and Measurement, 2012, 61 (6), pp.1719-1732. 10.1109/TIM.2012.2187246 . hal-00658278

\section{HAL Id: hal-00658278 \\ https://hal-centralesupelec.archives-ouvertes.fr/hal-00658278}

Submitted on 10 Jan 2012

HAL is a multi-disciplinary open access archive for the deposit and dissemination of scientific research documents, whether they are published or not. The documents may come from teaching and research institutions in France or abroad, or from public or private research centers.
L'archive ouverte pluridisciplinaire HAL, est destinée au dépôt et à la diffusion de documents scientifiques de niveau recherche, publiés ou non, émanant des établissements d'enseignement et de recherche français ou étrangers, des laboratoires publics ou privés. 


\title{
A Matched-Pulse Approach for Soft-Fault Detection in Complex Wire Networks
}

\author{
Layane Abboud, Student Member, IEEE, Andrea Cozza, Member, IEEE, and Lionel Pichon
}

\begin{abstract}
The idea of Matched-Pulse (MP) reflectometry is here introduced, as an improvement for fault-detection techniques in wire networks. As opposed to existing reflectometry methods, the testing signal is network-dependent and selfadaptive, and designed in a way as to maximize the probability of detection of an echo associated to an eventual fault. The detection performance of this novel technique is investigated by means of a mathematical analysis comparing it to standard Time-Domain Reflectometry (TDR) methods. It is proven that the more complex the network the better the performance the MP approach will provide. This is opposed to common understanding, as standard TDR techniques yield degrading detection probabilities in the case of the complex networks. Furthermore, the MP approach is shown to be particularly interesting for soft-fault detection, as it provides an improved performance as soon as the fault is weakly reflective. Our findings show that the MP approach is expected to bring a substantial improvement in the likelihood of properly detecting faults in configurations that are deemed critical for standard reflectometry techniques, particularly when dealing with noisy NUTs. Experimental results measured on coaxial complex networks support these claims.
\end{abstract}

Index Terms - Time-domain reflectometry, fault detection, soft faults, complex networks.

\section{INTRODUCTION}

W IRE networks are present in most modern systems, where the transmission of information and energy is crucial for their proper functioning [1]. We can mention transport systems, nuclear power plants, overland transmission lines, buildings, communication infrastructure, etc. The wide use of wires has made the problem of safety and reliability an important issue, provided that in many cases a faulty wire can cause the failure of a whole system [2], [3].

In a general way, wire faults are classified into two main categories [4], [5]: hard faults, which are mostly open and short circuits and lead to a strong modification in signal propagation along the network; and soft faults, which include minor faults, e.g., insulation damages crushed lines, water infiltration, etc., where the energy reflected is typically just a negligible fraction of the incident one. These latter affect signal propagation to a minor extent, but they can be reckoned to eventually develop into hard faults [1] [6], whence the importance of their early detection.

L. Abboud and A. Cozza are with the Département de Recherche en Électromagnétisme, Laboratoire des Signaux et Systèmes (L2S), UMR 8506 SUPELEC - Univ Paris-Sud - CNRS, 3 rue Joliot-Curie, 91192 Gif-sur-Yvette, France. Contact e-mail: andrea.cozzalsupelec.fr.

L. Pichon is with the Laboratoire de Génie Électrique de Paris (LGEP), UMR 8507 SUPELEC - Univ Paris-Sud - CNRS, 11 rue Joliot-Curie, 91192 Gif-sur-Yvette, France.
Although several testing methods have been developed during the last decades to detect and locate faults [1], reflectometry is one of the most widely applied [7]. A number of declinations of this approach have been proposed [4] [8] [9] [10] [11], mostly aiming at improving the localization capabilities of the technique by using specifically optimized testing signals; yet, reflectometry methods share the common idea of injecting testing signals into the network under test (NUT), and assess the presence of eventual faults by analyzing the signals reflected back by the network. On the one hand, hard faults such as short or open circuits react to the testing signal by reflecting most of its energy back: as such, they can be more readily detected and distinguished from other sources of reflection (e.g., junctions between several branches). On the other hand, soft faults often present very weak responses, with likely dispersive frequency responses, whence the intrinsical difficulty in their detection. Typical examples of soft faults are frays in the insulation of cables, damages in their coating and shielding, and any local modifications of their geometrical and/or electrical properties that do not hinder the propagation of energy through them in a substantial manner.

It has been shown that the detection of soft faults is difficult even in the simplest configurations [4] [12] [13], because the amplitude of the reflected wave, caused by this type of faults, can be in some cases comparable to the noise level, or other residual signals due to impedance discontinuities along the wire network. The case of more complex networks is by far harder: under the class of complex networks we will consider all network presenting at least one junction between different branches. More specifically, a complex NUT will be regarded as potentially capable of responding to a test pulse by means of a series of echoes. This type of configurations are more often encountered in embedded systems (e.g., vehicles), where the network may happen to be used for signal and power distribution, while less representative of power grids.

When dealing with complex networks the echoes caused by the presence of a fault are likely to be of the same (or an even weaker) order of magnitude as for the echoes coming from other parts of the NUT, has seemingly not received much attention so far, due to the intrinsical limitations of existing Time-Domain Reflectometry (TDR) methods [12]. To the best of our knowledge, there is no specific technique conceived to deal with the problem of soft-fault detection in complex wire networks. Such absence is surprising since, as we already recalled, soft faults can actually develop into hard ones. The early detection of soft faults is hence a critical issue in the development of reliable wire structures.

Current TDR techniques are based on the idea of testing 
the NUT by means of a specifically designed signal, be it pulsed, harmonic or an ad hoc signal, applied to an input port coupled to the NUT [12]. In this paper, we propose an alternative approach where the excitation signal is partly determined by the NUT itself, rather than being set beforehand. This idea, hereafter referred to as the Matched-Pulse (MP) approach, requires an additional experimental step with respect to standard TDR techniques. The following Sections will demonstrate that thanks to this approach the probability of detecting the presence of a soft fault in a critical scenario is systematically improved. Although it could be applied to any configuration, it provides practical advantages only within the framework of complex networks. By this term we refer to NUTs characterized by an impulse response involving a large number of echoes, rather than just a few reflections: it is indeed more common to consider NUTs with a limited number of echoes[4], [6], [8], [9], [10], [11], [12].

In this respect, the MP is complementary to current techniques: in particular, as signal propagation through a NUT becomes increasingly complex, standard TDR techniques are known to provide a worsening performance; it is remarkable, as shown later, that the benefits of the MP approach are actually boosted by the presence of complex propagation as recalled in Section II and formally demonstrated in Section III. The expected improvement is not surprising, since the MP approach inherits the properties of time-reversal-based techniques: as well known in acoustics, it thrives on media rich in multiple-scattering interactions, a situation very similar to the case of complex wire networks [14], [15], [16]. Therefore, it could be used as a true alternative solution in configurations where standard TDR techniques are just incapable of distinguishing between fault-generated echoes and other physiological spurious signals.

The ability of the MP approach in gaining some extra dBs in the signal-to-noise ratio (SNR) in the reflected signal may have a vital role in the detection of the onset of a fault that is not yet sufficiently strong (or hard) to be detectable by means of standard TDR techniques. Although the MP approach cannot lead to any improvement in the localization of faults with respect to standard TDR techniques, its superiority in the issue of fault-detection in critical scenarios where often no information at all can be obtained is a fundamental asset that should not be underestimated.

The results presented in this paper are based on the case of networks based on scalar transmission lines, e.g., coaxial cables, twisted pairs, etc. The rationale for this choice is the wide-spread use of this type of networks: as a matter of fact, it is rare to find a paper in the literature about fault detection not dealing with this type of transmission lines. Moreover, the simple analytical representation they command allows deriving general results that serve as a predictive tool in understanding the impact of the several parameters describing an NUT. For any other type of line based on multi-conductor transmission, a more complex approach should be required: although it can be shown that the optimal properties of the MP approach are maintained even in more complex scenarios, this topic is out of the scope of this paper, which is primarily intended for the introduction and analysis of this novel technique.
The paper is organized as follows: the ideas and motivations behind our proposal are presented in Section II, where the simple modification required for the application of the MP approach is described in detail; this procedure is then formally studied in Section III by means of a mathematical model, taking into account a very general configuration of multipleecho NUT. Dispersive responses are also considered. This Section is the core of our proposal as it allows understanding in a precise manner under what conditions the MP approach can be deemed capable of providing any improvement with respect to standard TDR techniques. A number of results is thus predicted, and it is in particular shown that the MP cannot in any way provide any benefits when considering the case of NUTs with a response limited to just a few echoes, thus restricting its domain of application to the case of complex NUTs. The analysis presented in Section III is also applied to the problem of estimating the probability of correct detection of a fault, a fundamental asset in any applications requiring a decision about the detection of an event (a fault, here). Section IV finally presents experimental results supporting our conclusions about the improved detection capabilities of the proposed procedure.

\section{THE MP APPROACH}

The idea behind the MP approach was inspired by target detection in radar for a cluttered environment where a number of reflective objects surround the actual target [17]. Within this context, it was demonstrated [18], [19] that by using the properties of time-reversal signal processing [20], the return echo from the target can be maximized, while using the same amount of energy for the excitation signal. What is even more important is that the contrast between the signals related to the actual target and the clutter signals appears to be stronger with this approach than with any other technique. This property is demonstrated within the framework of fault detection in Section III.

A close relationship clearly exists between the problem of target detection in radar and fault detection in wire networks. Although propagation is guided in the latter, propagation and scattering phenomena can be described by similar laws in both cases. Fault detection fundamentally aims at drawing a picture of the propagation scenario seen through an electrical input port, whereas radar aims at doing the same but with antennas in open media. In both cases, the excitation signal is used as a proxy for imaging the presence of scatterers. If in radar these are typically point scatterers, in fault detection they take the shape of impedance discontinuities, such as junctions between the branches of a wire network, impedances representing circuits connected to the network or localized faults. Since in complex networks a large number of such discontinuities is common, multiple reflections from them provide a scenario very similar to a cluttered medium. Eventual faults thus play the role of targets whose presence must be detected with a high probability against spurious echoes due to the cluttered environment.

Although the above-mentioned references do not provide a physically-based justification of their remarkable features, 


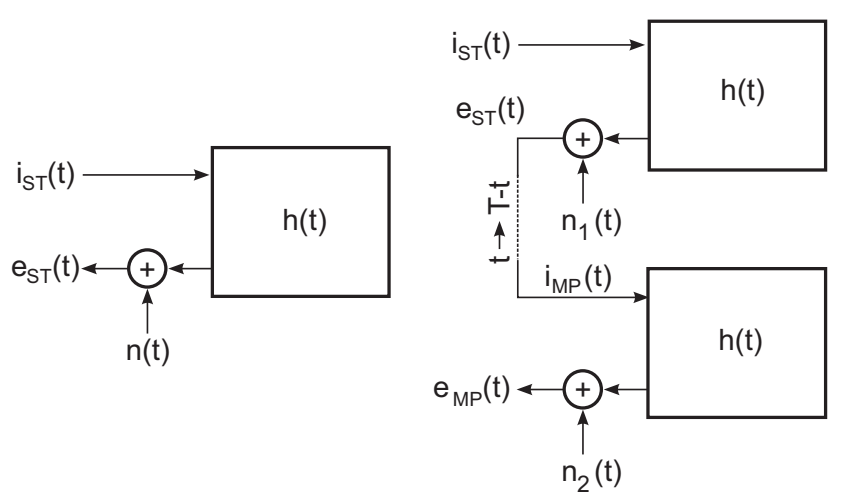

(a)

(b)

Fig. 1. Block-diagram representations for: (a) standard TDRs and (b) MP methods. The reflectometry impulse response of the NUT is noted as $h(t)$, whereas the $n_{i}(t)$ signals are equivalent additive noise sources at the output of the NUT. The time-delay $T$ is the overall duration of the standard TDR test.

the experimental results they present make up an interesting enough case for their transposition to the context of fault detection. Whence the idea of applying to fault-detection problems the concepts originally developed by using timereversal signal processing in radar detection, as presented for the first time in [21].

The procedure used to obtain such excitation signals is described in the literature [20] and is schematically represented in Fig. 1. Figure 1a represents the test of a NUT by means of a standard TDR approach. The injected signal (i.e., the testing signal) in time domain is noted as $i_{\mathrm{ST}}(t)$. The reflected signal, i.e., the standard TDR echo, is denoted as $e_{\mathrm{ST}}(t)$. This is the signal that in any standard TDR technique, after being properly post-processed, eventually allows detecting and locating a fault.

In the MP case, illustrated in Figure $1 b, e_{\mathrm{ST}}(t)$ is no more the ending point of the test, but it just provides an intermediary step. In fact, we define our testing signal, or MP, as the timereversed version of the standard TDR echo. The MP testing signal, referred to as $i_{\mathrm{MP}}(t)$, is the actual testing signal and is thus injected into the NUT so as to obtain the MP echo $e_{\mathrm{MP}}(t)$. This procedure does not require any assumption on the nature of the originally injected TDR signal $i_{\mathrm{ST}}(t)$ : as a matter of fact, it could be any of the testing signals developed within the context of other TDR techniques. It is important to acknowledge that the actual shape of the MP signal depends on the system's configuration, unlike other TDR techniques. The MP is clearly still a TDR technique, but with a new definition of the testing signal. Such an approach is next proven to result in a higher efficiency compared to the original testing signal $i_{\mathrm{ST}}$. But the fact that the starting point is the use of a standard TDR signal, implies that its properties are preserved when passing to the MP excitation. This could be useful when considering special excitation signals with high noise-rejection, such as spread-spectrum signals [9].

Some preliminary studies of the performance of the MP approach have been presented in [22], [21]. In this paper, we propose a more formal investigation and justifications for the improvement the MP ensures, while focusing on its impact on the probability of detection of a fault in a NUT.

\section{MATHEMATICAL ANALYSIS}

In order to evaluate the performance of the MP method, we propose to compare its performance against that of a standard TDR one. This comparison will be carried out in a formal way, by means of a mathematical analysis. To this end, we need to introduce a propagation model for the signal applied to a NUT. With no loss of generality, the impulse response of a typical NUT can be modeled as a superposition of time-delayed echoes, with varying amplitude depending on the sequence of interactions between the testing signal and the propagation discontinuities found along the NUT. These echoes are not merely meant to model reflections at junctions, but more generally any local discontinuity, e.g., local nonidealities in a transmission-line. In the rest of this Section, all of the signals will be assumed to be mathematical signals, i.e., expressed in squared-root of Watt units.

Since we are mainly interested to the case of soft-faults, TDR techniques are seldom directly applied to the NUT. More often, what can be referred to as the difference system is actually studied: by this term, we consider the difference of the responses obtained from the actual NUT and a reference one (or baseline) that is assumed to be without any fault. This procedure allows removing most of the physiological echoes of the NUT that are not related to any fault (e.g., reflections at junctions and far ends of branches), which constitute the clutter response hindering the detection of eventual faults: as a result, minor echoes potentially related to faults are more clearly exposed, leading to an improved detection dynamic [12], [23]. This procedure is practically inevitable as soon as the faults considered present a negligible reflectivity, hence leading to echoes from junctions much stronger than those from the fault.

For this approach to hold, time-invariance is an important requirement. This does not mean that the system is in a permanent steady-state: rather, that for the time between the two measurements leading to the difference response, the NUT has eventually changed only by the appearance of a fault. In some practical configurations, such as in TDR systems embedded into transportation systems, this assumption should be carefully considered, as mechanical vibrations can lead to spurious reflections due to non-perfect connections. Such a scenario is not considered in this paper, as it only represents a part of the systems to which TDR techniques are applied. More importantly, the aim of this paper is to assess whether under the same working conditions, testing signals generated through the proposed method are capable of improving the detection capabilities of TDR techniques.

Within this framework, the impulse response of the difference system relates the difference of the output signals reflected back by the networks (the one under test and the reference one) to the input test signal. As a result, the output signal will be made up by a series of echoes directly related to the reflectivity of the fault, hereafter referred to as $\Gamma_{F}(t)$.

No assumption will be formulated on the actual nature of the fault, but for the fact that it be indeed soft, i.e. when 
considering the fault alone, the energy it reflects back be just a fraction of the incident one, requiring

$$
\Gamma_{F, \mathrm{rms}}^{2}=\frac{1}{B_{T}} \int\left|\Gamma_{F}(\omega)\right|^{2} \mathrm{~d} \omega \ll 1,
$$

where $B_{T}$ is the region of Fourier spectrum occupied by the excitation signal, not necessarily a continuous region. The root-mean-square (rms) value $\Gamma_{F, \text { rms }}$ of the reflectivity of the fault is thus required to be weak enough to regard the fault as soft, thus acting as a weak perturbation in the propagation of signals across the NUT.

As a result, the overall impulse response of the NUT (with respect to a baseline) can be stated as

$$
h(t)=\Gamma_{F}(t) * \sum_{i=1}^{N} \alpha_{i} \delta\left(t-t_{i}\right),
$$

where $\delta(t)$ is the Dirac pulse and the star stands for the convolution operator. The impulse response is thus modeled as a sequence of echoes of relative amplitude $\alpha_{i}$ occurring at the time $t_{i}$, representing the resulting interaction with the remaining NUT discontinuities of the energy scattered by the fault and the eventual attenuation of the echoes due to propagation losses. Subsequent interactions of these echoes with the fault can be neglected, thanks to the weak-perturbation hypothesis. Typically, the sequence $\left\{\alpha_{i}\right\}$ is broadly geometric, with an overall exponential reduction of their amplitudes. In the rest of the paper, the sums will only be characterized by the index of summation, with no further details.

Although simplified, the model in (2) can actually represent the most important physical phenomena underlying the propagation of signals through a complex network. Not only the possibility of complex series of echoes is accounted for through the $\left\{\alpha_{i}\right\}$ sequence, but the eventual dispersive response of the fault is modeled by $\Gamma_{F}(t)$.

The aim of the subsequent study is twofold: 1) to support our claim of a stronger output signal reflected by the fault in increasingly complex configurations excited by means of MP signals; 2) to provide a quantitative treatment of the advantages of the MP method in terms of detection gain and detection probability in presence of a realistic NUT affected by an additive Gaussian noise.

\section{A. Detection gain}

In the following analysis, a standard TDR signal $i_{\mathrm{ST}}(t)$ will be considered, with no assumption made on its nature. As thus, the results here presented are entirely general and apply to any TDR method.

In order to properly compare the standard TDR and MP approaches, we are going to impose the same injected energy, the idea being of checking whether the shape of the MP signal is better suited at exciting a fault, in order to yield a higher output starting from the same amount of available injected energy.

The detection of a fault is usually based on the identification of the strongest signal reflected back by the NUT, after subtracting the baseline echo. In simple words, the output signal is analyzed looking for a contribution stronger than a given reference threshold suitably fixed in advance. This threshold is typically set as a compromise between the ability to detect the presence of an echo (and thus an eventual fault) and the need to provide for a robust immunity with respect to noise signals (see Section III-B). Independently from the actual type of testing signal, the use of a threshold-detection approach implies that the quantity of interest is the maximum instantaneous power of the echo. It is defined, for the case of a standard testing signal as

$$
\mathcal{P}_{\mathrm{ST}}^{\text {out }}=\max _{t}\left|e_{\mathrm{ST}}(t)\right|^{2}
$$

where $e_{\mathrm{ST}}(t)$ is the echo signal generated by applying a testing signal $i_{\mathrm{ST}}(t)$, as introduced in Section II. In simple NUT configurations, it is reasonable to expect this maximum to coincide with the first echo generated by the interaction with the fault; this allows retrieving the position of the latter. Actually, as it will be shown in the experimental results presented in Section IV, this assumption does not hold anymore in complex NUTs. Hence, peak-based detection schemes cannot systematically ensure the precise localization of the fault under such circumstances. This fact is shared by any standard TDR scheme, as well by the proposed approach.

The efficiency of the TDR scheme can thus be precisely measured by looking at the ratio $\mathcal{P}^{\text {out }} / \mathcal{E}^{\text {in }}$, where $\mathcal{E}^{\text {in }}$ is the energy of the testing signal applied to the NUT. Hence, the improvement provided by the MP approach over standard TDR signals can be assessed by introducing the following definition for the detection gain

$$
G=\frac{\mathcal{P}_{\mathrm{MP}}^{\text {out }} / \mathcal{E}_{\mathrm{MP}}^{\text {in }}}{\mathcal{P}_{\mathrm{ST}}^{\text {out }} / \mathcal{E}_{\mathrm{ST}}^{\text {in }}}
$$

where the quantities indexed by MP are defined as those with ST, but considering the signals generated by applying the MP test signal $i_{\mathrm{MP}}(t)$. The gain in (4) assesses whether the fault is more strongly excited by means of an MP testing signal with respect to a standard TDR one, having ensured that the same input energy is available for the test.

In order to calculate this gain, we first consider the output signal $e_{\mathrm{ST}}(t)$ obtained in the standard TDR case

$$
e_{\mathrm{ST}}(t)=i_{\mathrm{ST}}(t) * h(t)=\sum_{i} \alpha_{i} e_{\mathrm{ST}}^{o}\left(t-t_{i}\right),
$$

with

$$
e_{\mathrm{ST}}^{o}(t)=i_{\mathrm{ST}}(t) * \Gamma_{F}(t)
$$

the elementary response of each echo found in $e_{\mathrm{ST}}(t)$. The input energies in (4) are given by

$$
\mathcal{E}_{\mathrm{ST}}^{\mathrm{in}}=\int\left|i_{\mathrm{ST}}(t)\right|^{2} \mathrm{~d} t
$$

while for the MP approach

$$
\mathcal{E}_{\mathrm{MP}}^{\mathrm{in}}=\int\left|i_{\mathrm{MP}}(t)\right|^{2} \mathrm{~d} t=\int\left|e_{\mathrm{ST}}(T-t)\right|^{2} \mathrm{~d} t,
$$

where $T$ is the duration of the first phase during which $i_{\mathrm{ST}}(t)$ is applied to the NUT. For the sake of simplicity, we will drop the delay $T$, as it has no effect on our analysis. An 
acausal formulation will thus be considered. Inserting (5) into (8) straightforwardly yields

$$
\mathcal{E}_{\mathrm{MP}}^{\mathrm{in}}=\mathcal{E}_{o}\left[\sum_{i} \alpha_{i}^{2}+\sum_{i \neq j} \alpha_{i} \alpha_{j} \phi_{o}\left(t_{i}-t_{j}\right)\right]
$$

having introduced the energy $\mathcal{E}_{o}$ of the echo template $e_{\mathrm{ST}}^{o}(t)$ and its peak-normalized auto-correlation function $\phi_{o}(t)$.

Equation (9) is composed by two terms: the first one is given by the sum of the energy of each individual echo, as though they were not superposed, while the second one represents the sum of the mutual energy of each couple of echoes. When comparing these two terms, it appears that the first one is a coherent sum, more specifically a positive-term series, while the second one involves the cross-products $\alpha_{i} \alpha_{j}$. This means that the second part of (9) happens to be an incoherent sum, as the weights $\alpha_{i}$ are typically zero-average quantities. This last remark comes from the fact that in complex networks most of the echoes are generated by junctions, which have by definition a negative reflection coefficient: hence, these echoes will change sign after each interaction with any other junction in the NUT. Under these circumstances, the first term in (9) is bound to be much higher than the second one. The contrast between these two terms systematically increases, on average, as the number of echoes $N$ increases, thus implying that complex networks will support the following approximation

$$
\sum_{i \neq j} \alpha_{i} \alpha_{j} \phi_{o}\left(t_{i}-t_{j}\right) \ll \sum_{i} \alpha_{i}^{2} .
$$

In the context of the present analysis, the above simplification will be applied, for the sake of simplicity.

In order to compute the detection gain, we just need to calculate the maximum instantaneous power for the two test signals; we first consider the output signal for the MP case

$$
\begin{aligned}
e_{\mathrm{MP}}(t) & =i_{\mathrm{ST}}(-t) * h(-t) * h(t) \\
& =\mathcal{E}_{\Gamma_{F}} i_{\mathrm{ST}}(-t) * \phi_{\Gamma_{F}}(t) * \sum_{k} \beta_{k} \delta\left(t-t_{k}^{\prime}\right) \\
& =\mathcal{E}_{\Gamma_{F}} e_{\mathrm{MP}}^{o}(t) * \sum_{k} \beta_{k} \delta\left(t-t_{k}^{\prime}\right),
\end{aligned}
$$

where $\phi_{\Gamma_{F}}(t)$ is the peak-normalized auto-correlation function of the fault response $\Gamma_{F}(t)$ and $e_{\mathrm{MP}}^{o}=i_{\mathrm{ST}}(-t) * \phi_{\Gamma_{F}}(t)$ is the elementary response from the fault for the MP case. The term $\mathcal{E}_{\Gamma_{F}}$ is the energy of the $\Gamma_{F}(t)$ function. The Dirac's delta series based on the $\left\{\beta_{k}\right\}$ sequence is nothing else than the auto-correlation function of the original Dirac's delta series based on the $\left\{\alpha_{i}\right\}$ sequence, with

$$
t_{k}^{\prime}=t_{j}-t_{i}
$$

and

$$
\beta_{k}=\left\{\begin{array}{cc}
\sum_{i} \alpha_{i}^{2} & k=0 \\
\alpha_{i} \alpha_{j} & k \neq 0
\end{array} .\right.
$$

All of the $\beta_{k}$ terms (echoes) are characterized by zeroaveraged weights, but for the echo $\beta_{0}$; this latter can be easily shown to correspond to the time of arrival of signals directly interacting with the fault and to be by definition the strongest one among the $\left\{\beta_{k}\right\}$. (3)

The instantaneous output powers are then found by applying

$$
\mathcal{P}_{\mathrm{ST}}^{\text {out }} \simeq \alpha_{\max }^{2} \max _{t}\left|e_{\mathrm{ST}}^{o}(t)\right|^{2}
$$

with $\alpha_{\max }=\max _{i} \alpha_{i}$, while for the MP case

$$
\mathcal{P}_{\mathrm{MP}}^{\text {out }}=\max _{t}\left|e_{\mathrm{MP}}(t)\right|^{2} \simeq \beta_{0}^{2} \max _{t}\left|e_{\mathrm{MP}}^{o}(t)\right|^{2} .
$$

Using (7), (9), (14) and (15), (4) yields

$$
G=G^{\prime} G^{\prime \prime}
$$

with

$$
\begin{aligned}
G^{\prime} & =\sum_{i} \alpha_{i}^{2} / \alpha_{\max }^{2} \\
G^{\prime \prime} & =\frac{\mathcal{E}_{\Gamma_{F}} \mathcal{E}_{\mathrm{ST}}^{\mathrm{in}}}{\operatorname{E}_{o}} \frac{\max _{t}\left|e_{\mathrm{MP}}^{o}(t)\right|^{2}}{\max _{t}\left|e_{\mathrm{ST}}^{o}(t)\right|^{2}}
\end{aligned}
$$

The two gain terms in (16) account for specific features of the NUT: $G^{\prime}$ is directly related to the complexity of the NUT, especially its topology, independently from the nature of the fault, whereas $G^{\prime \prime}$ depends on the fault response and the nature of the excitation signal. In order to assess whether the MP approach improves the detection of a fault, these two terms need to be studied. The first term (17a) is by definition greater than one, while the behavior of the second one cannot be claimed to be identically greater than one. Its actual value strongly depends on the type of signals considered, but some insight can be gained by considering two special configurations: 1) a non-dispersive fault; 2) an excitation pulse with a flat Fourier spectrum over a generic bandwidth $B_{T}$, that can as well be divided over a number of sub-bandwidths. In the first case,

$$
\Gamma_{F}(t)=\Gamma_{F} \delta(t)
$$

leading to $G^{\prime \prime}=1$. Conversely, in the second case,

$$
\begin{aligned}
G^{\prime \prime} & =B_{T} \frac{\max _{t} \int_{B_{T}}\left|\Gamma_{F}(\omega)\right|^{2} \mathrm{e}^{+\mathrm{j} \omega t} \mathrm{~d} \omega}{\max _{t}\left|\int_{B_{T}} \Gamma_{F}(\omega) \mathrm{e}^{+\mathrm{j} \omega t} \mathrm{~d} \omega\right|^{2}} \\
& =\left(\frac{\Gamma_{F, \mathrm{rms}}}{\max _{t}\left|\left\langle\Gamma_{F}(\omega) \mathrm{e}^{+\mathrm{j} \omega t}\right\rangle\right|}\right)^{2}
\end{aligned}
$$

where the brackets stand for the average operator applied over the bandwidth $B_{T}$ of definition of the excitation signal, and having applied Parseval's and the convolution theorems. The integral at the numerator has its maximum value at $t=0$, thus coinciding with $\Gamma_{F, \text { rms }}$ as defined in (1), while an upper bound for the denominator is provided by Cauchy-Schwartz inequality

$$
\max _{t}\left|\int_{B_{T}} \Gamma_{F}(\omega) \mathrm{e}^{+\mathrm{j} \omega t} \mathrm{~d} \omega\right|^{2} \leqslant B_{T} \int_{B_{T}}\left|\Gamma_{F}(\omega)\right|^{2} \mathrm{~d} \omega
$$

whence the fact that $G^{\prime \prime} \geqslant 1$. This result does not imply any assumption on the nature of the fault; it is remarkable that the lower bound for $G^{\prime \prime}$ is attained in what are usually regarded as the best-working conditions, i.e., for a non-dispersive fault: 
in this case the MP approach already provides a gain $G^{\prime}$ dependent on the complexity of the response of the NUT, as the detection gain is maximized as soon as the energy reflected back by the NUT is dispersed in a long series of echoes, rather than being concentrated into a single one. In other words, the MP approach benefits from an increased complexity in the response of the NUT, as opposed to standard reflectometry methods.

In any other condition, for an excitation with a flat spectrum, an extra gain is ensured by the fact that a fault with a response that has a higher self-resemblance (stronger dispersion) will also enable a $G^{\prime \prime}>1$; such an outcome is actually more likely when dealing with soft faults rather than hard ones. Hence, these results prove that the proposed approach can be expected to ensure a useful improvement with respect to any kind of predefined excitation signals. It is clearly not that surprising to find out such improvement, as this procedure is very closely related to the idea of matched filter, hence our claim that the MP approach is optimal in the sense of maximizing the detection probability, as will shown in the next Section.

The only configuration where $G=1$ is what is usually regarded as the simplest and most optimistic scenario, i.e., a single echo from a non-dispersive fault, such as the case of an infinitely long (or impedance-matched) wire, with a single fault and no other discontinuities. Clearly, this outcome could have been anticipated from the very definition of the MP procedure detailed in Section II: if the fault is non-dispersive, the echo will be a perfect replica of the test signal. Injecting it again into the NUT will not provide any further modification, for these same reasons: hence the complete equivalence between the MP approach and the original TDR technique used for its definition. This configuration is clearly not interesting, as the MP will not be different in any detail from a standard TDR technique. Therefore, this case will not be studied any further, since in the present paper we are rather addressing the more compelling case of complex networks. Again, this points to the fact that the MP approach is indeed complementary to existing reflectometry techniques, and should be considered only in configurations where standard approaches fail.

These results have been derived under fairly general assumptions. Two main parameters of interest in practical scenarios are losses and a limited bandwidth. The first one is actually accounted for by the sequence $\left\{\alpha_{i}\right\}$ : as losses increases, the time-spread of this sequence decreases, thus leading to a reduction in $G^{\prime}$. It is noteworthy to remind that the definition of $G^{\prime}$ does not depend on the absolute value of the $\left\{\alpha_{i}\right\}$, but rather on their relative distribution. The MP approach will provide a gain as long as the NUT responds with a long series of echoes.

The reduction of the excitation bandwidth has a different effect, as it will make the approximation (10) less precise. As the bandwidth decreases it will be no more possible to neglect the mutual energy, i.e., the overlapping of the pulses. Including this extra-term in the computation of the gain will have a broadly decreasing effect, as the echoes will be no more resolved.

\section{B. Detection probability}

The benefits brought by the detection gain (16)-(17) are better perceived when recalling that real-life NUTs are always affected by some kind of background noise. Independently from its origin, the presence of noise imposes a strong limit to the dynamic-range of the signals that can be properly detected. In the case of soft faults, as their echoes can be expected to be quite weak, the possibility of having faultrelated echoes beneath the level of background noise is clearly a critical configuration. Within this context, the ability of the MP approach to yield a higher echo is thus a strong argument in its favor.

It is therefore important to assess how the detection gain translates into a corresponding improvement of the detection probability. As such, it is convenient to compute the SNR of the output signal at the instant of the detection, i.e., when it reaches its maximum amplitude: this is a fundamental measure of the reliability of the output signal obtained from a reflectometry measurement.

We consider an analysis based on the presence of an additive Gaussian noise. This choice does not bring any limitation to our conclusions, as the same analysis could be carried out considering other types of noise. The choice of an additive Gaussian noise is mainly motivate by the simplicity of the related analysis and its widespread observation in real-life NUTs.

We start off with the case of a standard TDR approach, as depicted in Fig. 1(a). As the decision of whether a fault is present or not directly depends on the SNR at the time of arrival of what is assumed to be the fault-related echo, we focus our analysis on this quantity. In particular, we consider that the original testing signal $i_{\mathrm{ST}}(t)$ be pulse-like: in this way, the SNR can be defined as follows:

$$
S_{\mathrm{ST}}=\frac{\mathcal{P}_{\mathrm{ST}}^{\text {out }}}{2 N_{0}}
$$

i.e., as the ratio between the peak instantaneous power of the fault-related echo (i.e., the one related to the coefficient $\alpha_{\max }$ for standard TDR techniques), and the noise average power. The average power for the actual noise applied to the output port is set to $N_{0}$, while the factor 2 results from the use of the difference output signal, since two noise realizations intervene in its measurement.

In the case of TDR techniques based on test signals shaped otherwise than pulses, particularly when the output signal is actually correlated to the injected one before proceeding to the detection analysis (e.g., spread-spectrum TDR [9] or timefrequency techniques [6]), the same definition still applies. Rather than the instantaneous power, the energy of the signals should be considered, but no major difference will be required. A similar consideration can be invoked for the noise average power: the average energy after filtering through the correlator will be considered. Indeed, in the case of correlation-based TDRs, pulse-like sequences can still be expected at the output of the correlator between the output TDR signal and the template one.

With reference to Fig. 1(b), we now turn our attention to the MP case. Now, the output signal from the first step of 
standard TDR is already corrupted by the Gaussian noise; as its time-reversed version is injected back through the NUT, the output signal for the MP will be corrupted by two noise terms, the one from the first step and then again at the output of the MP signal. Hence, the overall equivalent noise $n_{\mathrm{eq}}(t)$ can be expressed as follows

$$
n_{\mathrm{eq}}(t)=n_{1}(-t) * h(t)+n_{2}(t)
$$

where $n_{1}(t)$ and $n_{2}(t)$ are defined in Fig. 1(b). It is important to acknowledge the fact that in the MP approach the noise $n_{1}(t)$ is scattered back by the NUT generating a series of delayed and scaled replicas, filtered by the fault response, acting as a number of practically uncorrelated noise sources. The average power of the resulting equivalent noise can be proven to be

$$
N_{\mathrm{eq}} \simeq N_{0}\left(1+\Gamma_{F, \mathrm{rms}}^{2} \sum \alpha_{i}^{2}\right)
$$

whence

$$
S_{\mathrm{MP}}=\frac{\mathcal{P}_{\mathrm{MP}}^{\text {out }}}{2 N_{\mathrm{eq}}},
$$

Introducing the SNR gain $R$ defined as the perceived increase in the output SNR while the input energy is unmodified and recalling (4), we obtain

$$
R=\frac{S_{\mathrm{MP}} / \mathcal{E}_{\mathrm{MP}}^{\mathrm{in}}}{S_{\mathrm{ST}} / \mathcal{E}_{\mathrm{ST}}^{\mathrm{in}}}=\frac{G}{1+\Gamma_{F, \mathrm{rms}}^{2} \sum_{i} \alpha_{i}^{2}} .
$$

The sum at the denominator can be expected to reduce the gain in the SNR brought in by the MP approach. In fact, the second term in it is the fraction of energy reflected back by the NUT, when excited by a flat-spectrum testing signal. As the fault is passive, this fraction of energy is clearly bound to be smaller than one. Hence

$$
G \geq R \geq \frac{G}{2}
$$

The lower-bound is only met in the trivial case of a nondispersive hard fault with a single echo $\left|\alpha_{1}\right|=1$. In all other cases, and particularly for the case of soft faults, the sequence of $\left\{\alpha_{i}\right\}$ would lead to a higher gain allowing to fully take advantage of the higher detection gain yielded by the MP approach. Some insight is provided by the special case of a NUT where neither dissipation phenomena nor dispersive ones occur, not even at the loads. This ideal case involves

$$
\sum_{i} \alpha_{i}^{2} \equiv 1
$$

due to energy conservation. Whence

$$
R=\frac{1}{\alpha_{\max }^{2}\left(1+\Gamma_{F, \mathrm{rms}}^{2}\right)} \simeq \frac{1}{\alpha_{\max }^{2}},
$$

due to the hypothesis (1). This result implies again that the MP approach cannot lead to any advantage whenever the whole energy reflected back by the NUT is concentrated into a single echo, whereas $R$ can be much higher than one as soon as the energy associated to the strongest echo only represents a small portion of the overall output energy, e.g., for a long sequence of echoes. This is indeed the configuration we are interested in.

Having derived a relationship between the SNR in the case of a standard and an MP approach, the SNR can be easily related to the detection probability by applying standard tools from decision theory [24], [25]. In particular, for the case of a Gaussian noise, given a decision threshold $a_{\text {th }}$ expressed as a fraction $0<\rho<1$ of the peak echo, plus the noise average power and the SNR, the probability of detecting an existing fault can be expressed as

$$
P^{\mathrm{TP}}=\frac{1}{2} \operatorname{erfc}((\rho-1) \sqrt{S}) .
$$

This is the conditional probability of detection, having imposed that the fault exists; this quantity is also referred to as true-positive (TP) probability of detection. Conversely, the false alarm rate, formally known as the false positive (FP) probability [26] $P^{\mathrm{FP}}$ is given by

$$
P^{\mathrm{FP}}=\frac{1}{2} \operatorname{erfc}(\rho \sqrt{S}) .
$$

The above expressions establish that the MP approach can ensure the same probability of detection of a standard TDR technique, but with a less stringent requirement for the SNR. More specifically, (25) implies that the MP approach can provide the same performance than a standard TDR technique while the noise average power is increased by a factor $R$. The other way round, for a given SNR, the MP can be expected to lead to a substantial improvement of the detection probability, depending on the type of response of the NUT. Remarkably, this improvement is expected to increase with the NUT complexity and the dispersiveness of the fault, a fact that is counterintuitive within the framework of standard TDR techniques.

\section{EXPERIMENTAL RESULTS}

In this section, we validate all the discussed ideas through experimentations. Some preliminary parametric investigations were previously conducted by means of numerical simulations, validating the idea of a detection gain increasing with the NUT complexity; these results are accessible in previous publications, e.g., [21] and deal with ideal non-dispersive faults. This is not the only reason for our focusing on experimental tests: our interest in soft dispersive faults would call for precise equivalent models that are not, to the best of our knowledge, easily accessible in the available literature. Experimental validations present the advantage of avoiding this difficulty, while also allowing to avoid any simplifying assumption in our setup.

It is noteworthy that the results here presented are not meant as a validation of the results derived in Section III. This latter is in fact self-contained and founded on grounds robust enough to provide a clear demonstration of the performance of the MP approach. The following results are rather meant as a practical demonstration of the capabilities of the proposed approach in a few test configurations. Inevitably, these examples cannot cover the entire range of possible combinations of NUTs found in practice. General results are provided in Section III. 


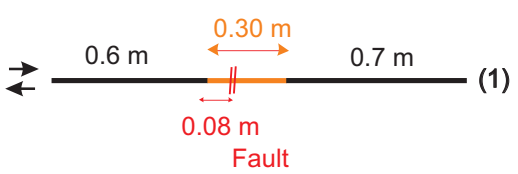

(a)

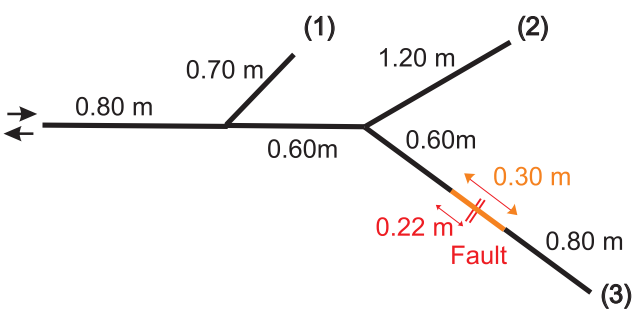

(c)

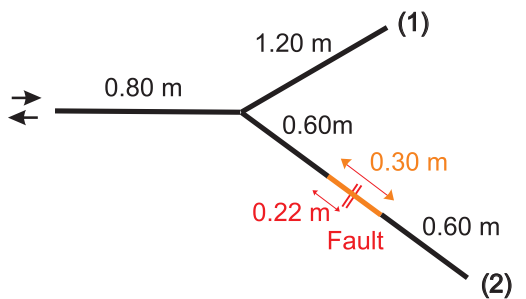

(b)

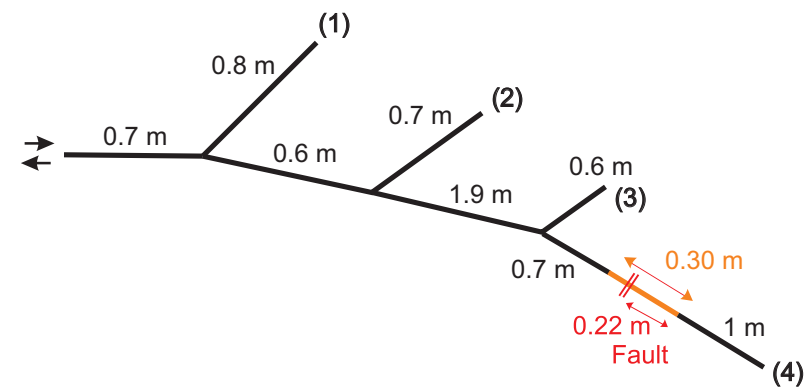

(d)

Fig. 2. Layouts of the NUTs considered for the experimental validation: (a) a single-branch line; (b) a single-Y junction; (c) a double-Y one and (d) an NUT with 3 junctions. Each branch of the NUTs consisted of a flexible coaxial cable with a $50 \Omega$ characteristic impedance, while the red portions represent the samples containing the fault, as shown in Fig. 4. The termination of the lines were left open-circuited or matched, depending on the test configuration, and are detailed in Table I, while the junctions were implemented by means of BNC T-junctions. The double-arrow symbol stands for the port at which the testing signals were applied.

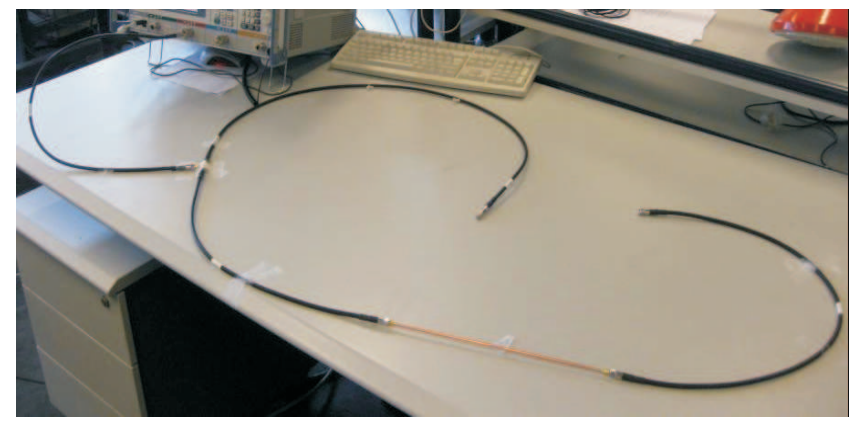

(a)

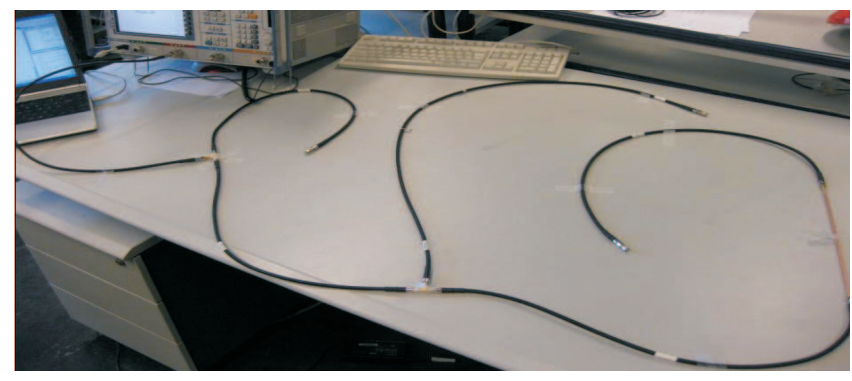

(b)

Fig. 3. Practical implementation of the single-Y and double-Y NUTs described in Fig. 2(b)-(c), as connected to a vector network analyzer for experimental tests. The $30-\mathrm{cm}$ long semi-rigid cable implementing the fault sample is clearly visible.

\section{A. Measurement setup}

As pointed out earlier in this paper, simple network configurations are very often tested in papers dealing with novel TDR schemes, such as a single-stretch of a damaged coaxial cable [6], [23], whose echo-impulse response is likely limited to a single echo. Under these conditions, the MP approach cannot provide any improvement in the probability of detecting a fault, as proven in Section III. For this reason, in this Section we considered a number of NUTs presenting very different echo-impulse responses, in order to validate our claim that for an increasing complexity, i.e., a larger number of echoes, the MP approach provides an improved detection probability, through a higher SNR of the reflectogram. A note of caution is necessary: the increasing number of Y-shaped junctions should not be mistaken for a gauge of a more complex response. As also shown later in this Section, depending on the type of impedance terminations, the same NUT can present a very different behavior. Even complex topologies such as that in Fig. 2(c) can yield just a few echoes as soon as each line is terminated by a matched load.

The NUTs considered during our tests are shown in Fig. 2, with two examples of their implementation provided in Fig. 3. Scalar transmission lines were considered, as often done in the analysis of TDR schemes, since this choice provides a simple and easily repeatable setup.

The need for a baseline measurement carried out without any fault was satisfied by resorting to a removable section in the NUTs, the red portion in Fig. 3. This 30-cm section consisted of a semi-rigid coaxial cable terminated by two SMA connectors; several identical samples of this structure were 


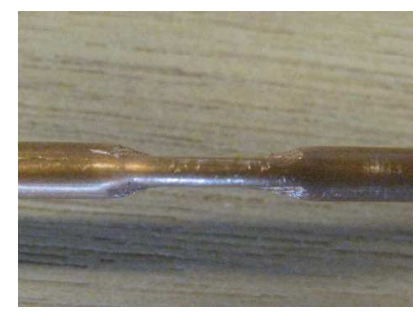

(a)

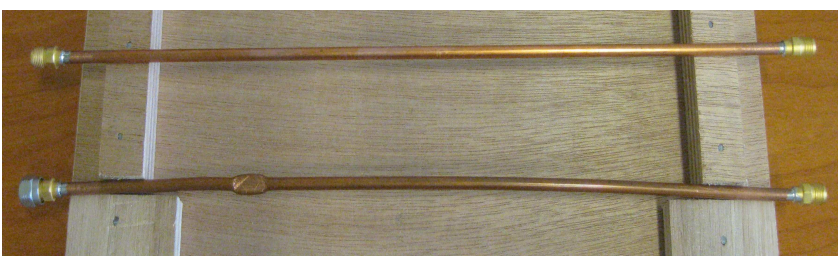

(c)

Fig. 4. The soft fault considered in the experimental validations, as obtained by crushing a $1-\mathrm{cm}$ long portion of a semi-rigid cable. The three pictures present: (a) a side view of the fault, with the cable reduced to a $2 \mathrm{~mm}$ thickness; (b) a front view of the fault; (c) the two samples used for the measurements, i.e., the reference semi-rigid cable without fault and the one presenting the fault. They share the same dimensions and features.

available, with one unaltered sample acting as the reference configuration and the others variously affected by minor faults. In the following, we will focus on the case depicted in Fig. 4, as explained later. The measurements thus consisted of a twostep procedure where the reference sample was connected in place in the first measurement, and subsequently substituted by the one with the fault in a second measurement. This approach was devised in order to ensure a repeatable setup.

The echo-impulse response of each configuration was measured by means of a vector network analyzer connected to the testing port. Actually, this rather means accessing the Fourier-spectrum of the impulse response; for this reason, the experimental results were post-processed by applying an inverse Fourier transform. The measurements were carried out over the frequency range from $300 \mathrm{kHz}$ to $8 \mathrm{GHz}$, with a intermediate-frequency filter bandwidth set to $100 \mathrm{kHz}$ : this ensured a strong rejection to noise, yielding very clean results that can be regarded as practically unaffected by any noise source. The input power was set to a $0 \mathrm{dBm}$ harmonic excitation signal.

These data characterize the entire behavior of the NUT, since they represent its transfer function: all of the quantities we have introduced in the previous Section can therefore be retrieved by means of straightforward post-processing techniques. In particular, the response of a NUT to any testing signal can be obtained by convolving the desired input signal with the experimentally measured impulse response of the NUT. This approach is clearly sound as the system under test is linear and time-invariant. Its main advantage is the simplicity with which we can obtain the responses of the NUTs to any signal, without needing an arbitrary waveform generator.

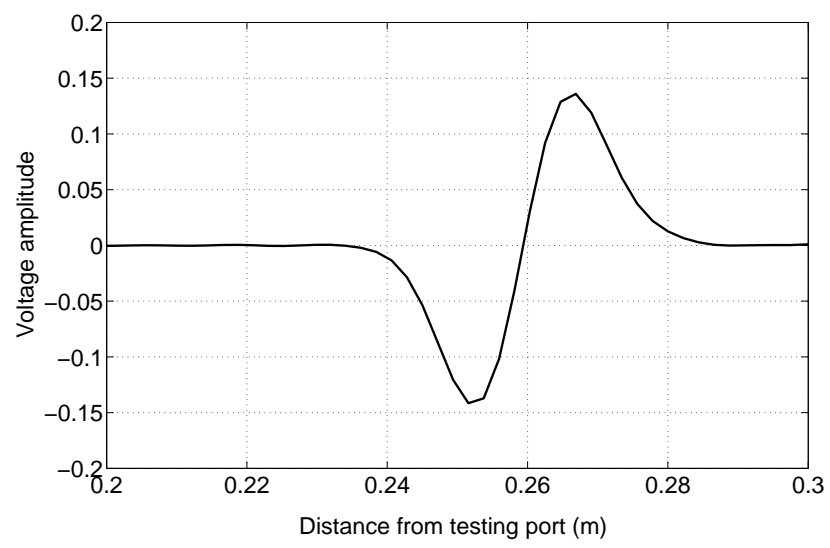

Fig. 5. The response of the fault shown in Fig. 4, as excited by a baseband Gaussian pulse with a $2 \mathrm{GHz}-3 \mathrm{~dB}$ bandwidth; the excitation signal was normalized to its peak amplitude. This response proves that the fault is indeed weakly reflective, with a derivative response, i.e., frequency-dependent or dispersive.

Moreover, as we are interested in studying the SNR of the output signals, it is easier to sum a synthetic additive Gaussian noise to the output signal during this post-processing phase than to actually apply it to the NUT. Clearly, this approach does not hinder the validity of our conclusions.

Being interested in soft faults, we focused our tests on the case of a crushed transmission line, as shown in Figs. 4a-b. Although other types of soft faults were previously considered in the literature, e.g., the case of a two-wire line with its dielectric coating partially removed [27], we have rather chosen the case of a crushed line as more realistic for the case of coaxial cables, since the removal of the dielectric medium is less likely. As a matter of fact, the configuration presently considered in this paper is analogous to the case of a local modification in the cross-section of a two-wire line (increased or decreased distance between the conductors), leading to a modification in the per-unit-length parameters of the transmission line. As such, this default is fairly general and can be regarded as representative for several types of transmission lines. The response of this type of fault was measured and is shown in Fig. 5, proving its weakly-reflective and dispersive nature, with a peak reflectivity of about $14 \%$.

\section{B. Results}

A grand-total of 8 configurations, detailed in Table I, were tested and the resulting impulse responses are shown in Figs. 6-9. These results refer to the case of a Gaussian pulse excitation with a $-3 \mathrm{~dB}$ bandwidth of about $2 \mathrm{GHz}$. These results show how the response of the NUT is strongly dependent not only on the topology of the NUT, but also on its electrical configuration, particularly its terminations. Although the number of echoes is not very different in the case of NUTs with matched ends, it is indeed greatly enhanced in the case of open-circuited ends: under these conditions, even a simple network topology can yield a large number of echoes, as visible in Fig. 6(b), for a simple line terminated by an open circuit. 

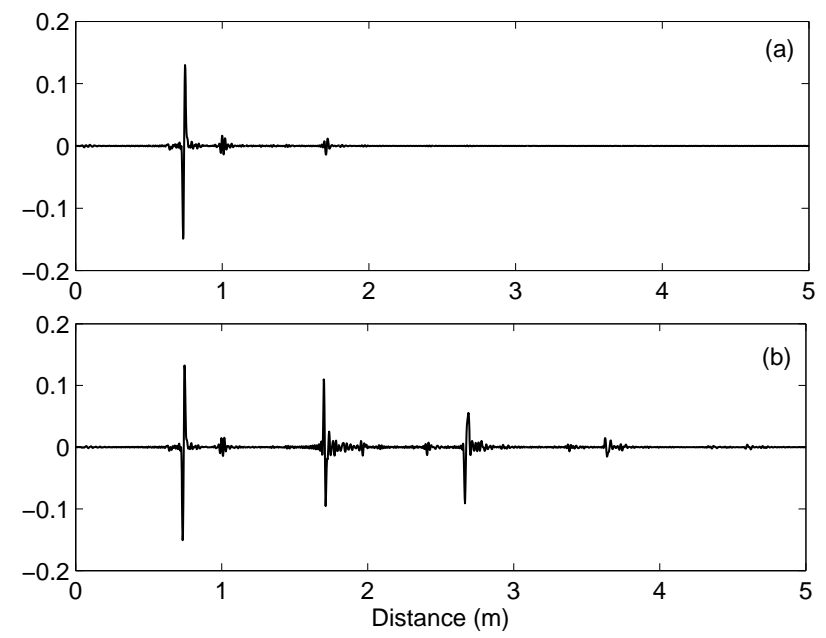

Fig. 6. The impulse responses for the (N1)-(N2) setups (simple line) shown in Fig. 2(a) and detailed in Table I.
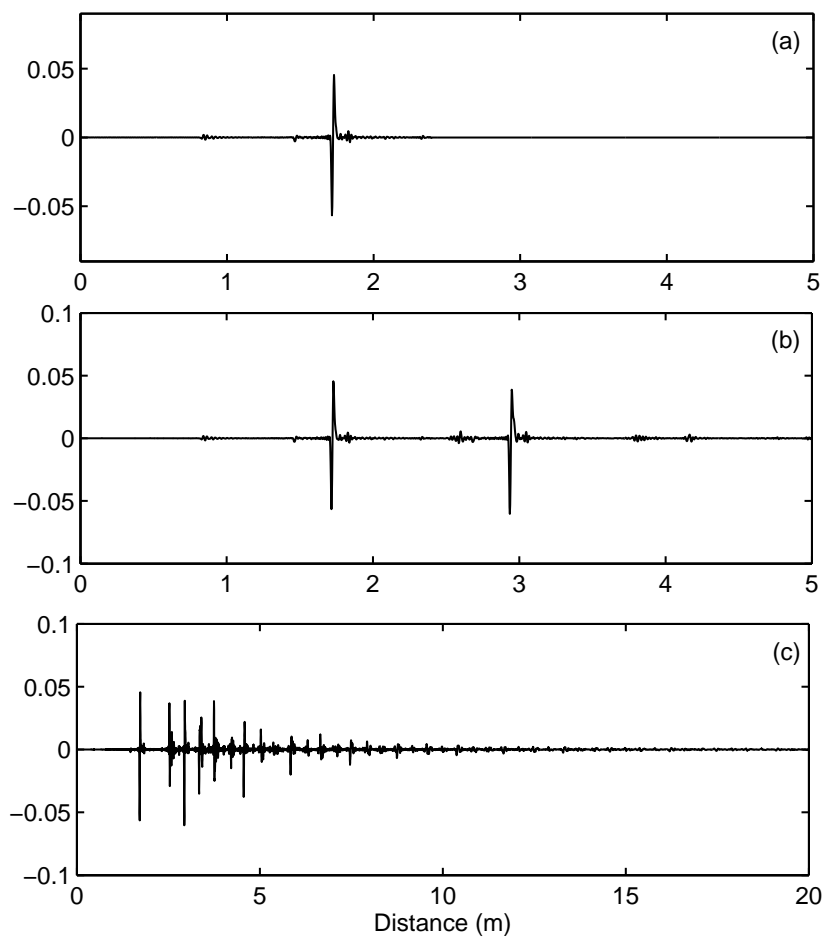

Fig. 7. The impulse responses for the (N3)-(N5) setups (single Y) shown in Fig. 2(b) and detailed in Table I.

According to (17), configurations with responses made up of just a dominant echo should be expected to yield a similar performance, with $G^{\prime} \simeq 1$ and a $G^{\prime \prime} \simeq 5.7 \mathrm{~dB}$. The MP would thus only provide a detection gain due to the fact that the energy of the testing signal would be concentrated around the frequencies at which the fault will respond, rather than being uniformly distributed over a predetermined bandwidth. Conversely, in the case of open-circuited ends $G^{\prime}$ is expected to provide a stronger contribution, due to a large number of echoes.
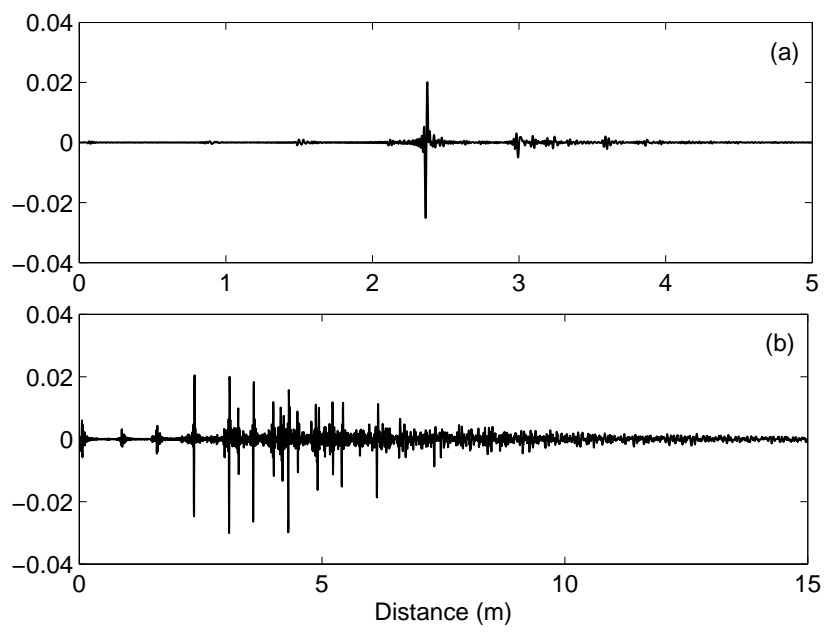

Fig. 8. The impulse responses for the (N6)-(N7) setups (double Y) shown in Fig. 2(c) and detailed in Table I.

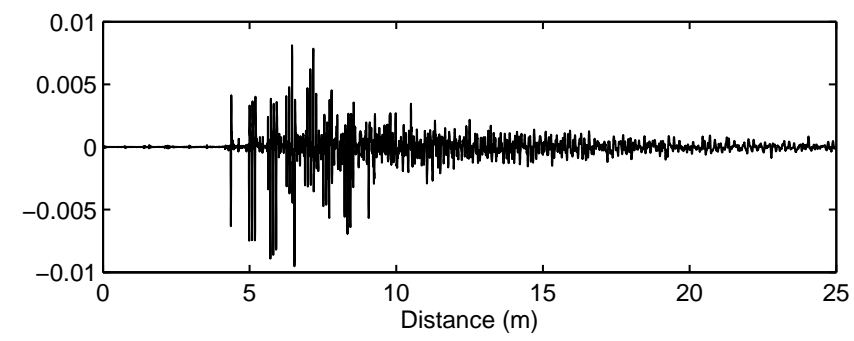

Fig. 9. The impulse responses for the (N8) setup (triple Y) shown in Fig. 2(d) and detailed in Table I.

These arguments are indeed supported by the results shown in Table II, where the ratio of the peak-value in the echoes obtained with a standard TDR testing signal and an MP one are summarized. The procedure described in Section IV-A was applied, by post-processing the measured impulse responses with the two testing strategies. It is important to recall that the fact of having used Gaussian pulses has no major effect on these results, as we have demonstrated in Section III that the detection gain is broadly independent on the choice of the standard TDR testing signal $i_{\mathrm{ST}}(t)$. Three different bandwidths were considered in these experiments, $200 \mathrm{MHz}$, $500 \mathrm{MHz}$ and $2 \mathrm{GHz}$. These differences were intended to assess how a changing bandwidth impacts on the performance of the MP approach. The results in Table II show that the bandwidth has a negligible effect on the cases of matched ends, with a typical variation of less than $1 \mathrm{~dB}$ on the gain, whereas for the other cases wider variations are observed. This outcome implies that the approximation (10) is actually valid only in configurations where the distance between the echoes is large enough as to make them distinguishable, even after having reduced the bandwidth, i.e., with a wider time-support for the excitation signal.

Table II also presents the detection gain predicted by means of (17), by taking the value of the peaks in the impulse responses equal to the $\left\{\alpha_{i}\right\}$ sequence. The gain $G^{\prime}$ estimated in this way is thus an upper-bound to the realized gain, since the 


\begin{tabular}{|c|c|c|c|c|c|c|}
\hline \multirow{2}{*}{ Ref. } & \multirow{2}{*}{ NUT type } & \multicolumn{4}{|c|}{ NUT terminations } & \multirow{2}{*}{$h(t)$} \\
\hline & & (1) & (2) & (3) & (4) & \\
\hline (N1) & Single lin & $\mathrm{M}$ & - & - & - & Fig. 6(a) \\
\hline (N2) & Single line & $\mathrm{O}$ & - & - & - & Fig. 6(b) \\
\hline (N3) & & $\mathrm{M}$ & $\mathrm{M}$ & - & - & Fig. 7(a) \\
\hline (N4) & Single Y & $\mathrm{O}$ & M & - & - & Fig. 7(b) \\
\hline (N5) & & $\mathrm{O}$ & $\mathrm{O}$ & - & - & Fig. 7(c) \\
\hline (N6) & & $\mathrm{M}$ & $\mathrm{M}$ & $\mathrm{M}$ & - & Fig. 8(a) \\
\hline (N7) & Double Y & $\mathrm{O}$ & $\mathrm{O}$ & $\mathrm{O}$ & - & Fig. 8(b) \\
\hline (N8) & Triple Y & $\mathrm{O}$ & $\mathrm{O}$ & $\mathrm{O}$ & $\mathrm{O}$ & Fig. 9 \\
\hline
\end{tabular}

TABLE I

CONFIGURATIONS OF THE NUTS CONSIDERED DURING THE EXPERIMENTAL TESTS. THE ELECTRICAL TERMINATIONS APPLIED AT THE ENDS OF THE NUTS SHOWN IN FIG. 2 ARE PROVIDED, WITH AN 'M' FOR

AN IMPEDANCE-MATCHED TERMINATION OR AN 'O' FOR AN OPEN

CIRCUIT. REFERENCES TO THE FIGURES SHOWING THE ECHO-IMPULSE RESPONSES OF EACH CONFIGURATION ARE GIVEN IN THE LAST COLUMN.

\begin{tabular}{|c|c|c|c|}
\hline \multirow{2}{*}{ Ref. } & \multicolumn{3}{|c|}{ Detection gains } \\
\cline { 2 - 4 } & $200 \mathrm{MHz}$ & $500 \mathrm{MHz}$ & $2 \mathrm{GHz}$ \\
\hline (N1) & $5.7(5.9)$ & $6.0(6.0)$ & $5.7(5.8)$ \\
(N2) & $8.1(8.4)$ & $7.7(8.6)$ & $8.4(8.7)$ \\
(N3) & $6.1(6.3)$ & $5.7(6.0)$ & $6.4(6.5)$ \\
(N4) & $7.2(7.5)$ & $8.4(8.2)$ & $8.2(8.2)$ \\
(N5) & $11.5(12.0)$ & $10.6(10.9)$ & $13.8(14.2)$ \\
(N6) & $5.1(5.7)$ & $6.3(6.4)$ & $6.5(6.6)$ \\
(N7) & $19.4(19.4)$ & $12.9(13.0)$ & $16.8(17.1)$ \\
(N8) & $11.6(12.2)$ & $13.2(14.5)$ & $17.0(17.2)$ \\
\hline
\end{tabular}

TABLE II

DETECTION GAINS, EXPRESSED IN DB, OBSERVED FOR THE EXPERIMENTAL CONFIGURATIONS INTRODUCED IN SECTION IV-A AND TABLE I, FOR THREE DIFFERENT EXCITATION BANDWIDTHS. THE VALUES IN PARENTHESIS ARE THE GAINS ESTIMATED BY APPLYING (16) AND (17) TO THE ECHOES OF THE MEASURED ECHO-IMPULSE RESPONSES $h(t)$, HAVING ESTIMATED $G^{\prime \prime}=5.7$ FOR THE FAULT SHOWN IN Fig. 4.

inevitable existence of overlapping peaks cannot be accounted for. Concerning the gain $G^{\prime \prime}$, it was estimated to be equal to $5.7 \mathrm{~dB}$, independently from the testing bandwidth, as required by its derivative nature.

As predicted by the arguments introduced in Section III, the gain is indeed almost independent of the network topology in the case of matched ends, where the impulse response is poor in number of echoes, whereas the MP ensures a strongly enhanced performance in the remaining configurations. These results are not based on a predictive model, but they are rather realized gains observed on the peak-value of the echo responses. As previously required, the energy of the testing signals was identical in all of the configurations.

The results presented in Table II can be compared to the number of echoes appearing in the impulse responses $h(t)$. The result of this operation is Fig. 10, where the correlation between the number of echoes in the impulse response and the detection gain of the MP approach is neatly exposed. The model derived in Section III allows stating that this correlation is in fact due to a causality relationship, as exemplified in (17). It is therefore not the topological complexity of the NUT that counts, but just the number of echoes in the impulse response.

\section{Reflectograms and detection probabilities}

It is interesting to study in more details the echo-responses obtained with a standard TDR and an MP approach. To

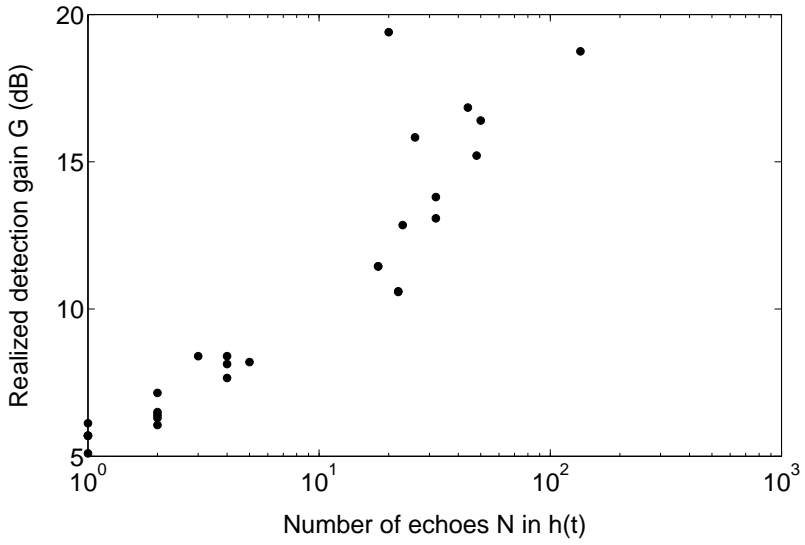

Fig. 10. Realized detection gain versus the number of echoes observed in the echo-impulse responses $h(t)$, by considering only the echoes with an amplitude at least one-tenth of the peak echo. The gains are given in Table II.

this end, we will focus in the rest of this Section onto two NUTs, the single-Y and double-Y structures, with all their ends terminated by open circuits. A bandwidth of $500 \mathrm{MHz}$ will be considered, and the testing signals will be set to an energy equal to one. The impact of the detection gain will thus be considered in the case of a noisy NUT, jointly with the predicted improvement on the detection probability.

The case of the single- $Y$ structure is first considered. Fig. 11(a) shows that the MP fault-related peak is about 3.4 times higher than the one obtained with the Gaussian-pulse excitation. Moreover, it also appears that the main peak of the MP approach is about 1.7 times higher than any other echo; conversely, the standard Gaussian TDR presents a fault-related echo that is actually 1.4 times weaker than the subsequent ones. Hence, also a standard TDR approach will not be able to correctly locate the position of the fault, as the most likely echo that will be detected is the maximum one about $3 \mathrm{~m}$ away from the testing port, rather than at $1.62 \mathrm{~m}$. The MP approach is therefore not only providing a remarkable improvement in the echo-response with respect to a standard TDR technique, but also a clearer result to interpret, with a single dominant echo featuring a fair contrast with respect to the remaining echoes.

Having access to the impulse response of the NUT, the detection gain $G$ can be estimated quite easily, by truncating the sum $\sum_{i} \alpha_{i}^{2}$. Considering just the first eleven echoes in (17a), we obtained a lower-bound estimate of $G^{\prime} \geq 7.27 \mathrm{~dB}$. This value is consistent with the results shown in Fig. 11(a), thus validating the prediction capabilities of (17) and the analysis presented in the previous Section, particularly the approximation (10).

The results in Fig. 11(a) imply that for the same level of background noise, the detection probability must be expected to be substantially higher with the MP approach. This improvement can be better assessed looking at the results proposed in Fig. 11(b), where an additive Gaussian noise was added during the post-processing to the output signals from the two TDR techniques: as expected, the detection gain provided by the MP approach yields an output signal where the presence of a 


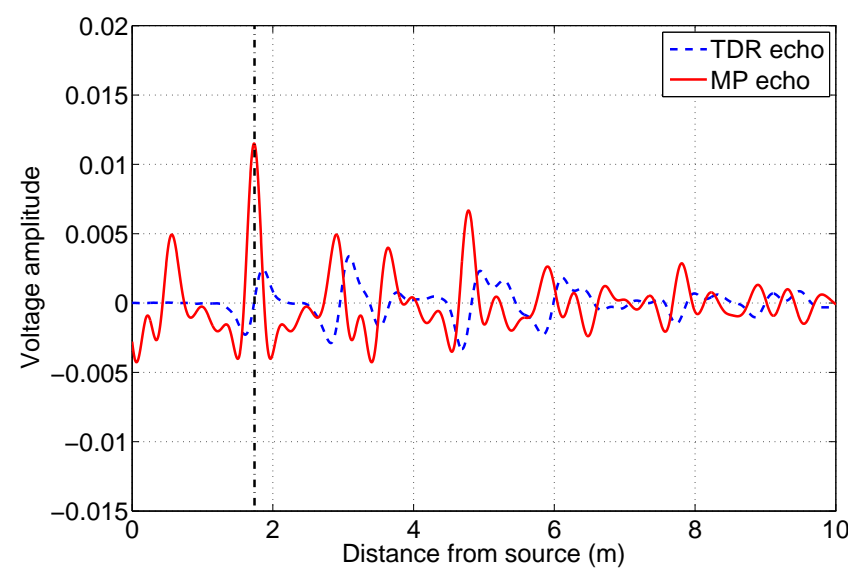

(a)

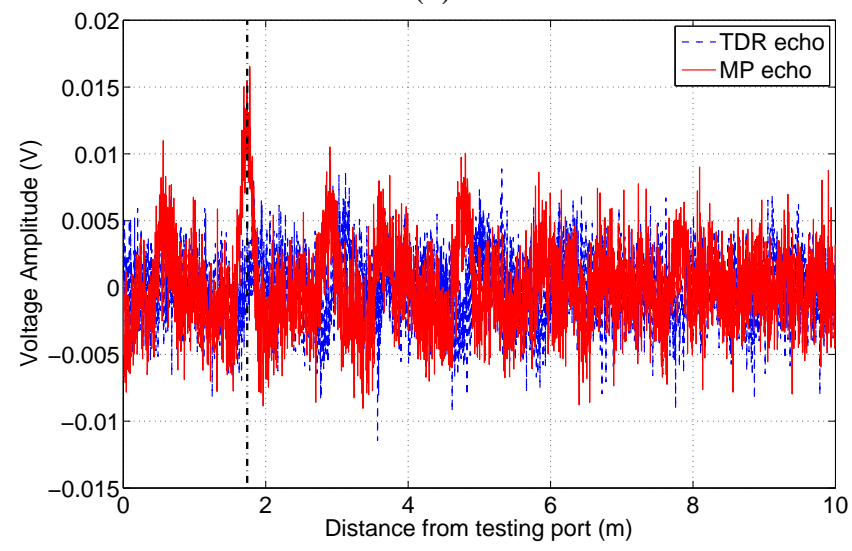

(b)

Fig. 11. The output signals obtained when exciting the network of Fig. 2(a) with open-circuited ends by means of a Gaussian pulse or the associated MP for: (a) a noiseless configuration and (b) the presence of an additive Gaussian noise signal with a fixed average energy, resulting in an SNR of $0 \mathrm{~dB}$ for the Gaussian TDR case, corresponding to an SNR of $10.6 \mathrm{~dB}$ for the MP one. The vertical dashed line represents the position of the fault-related echo.

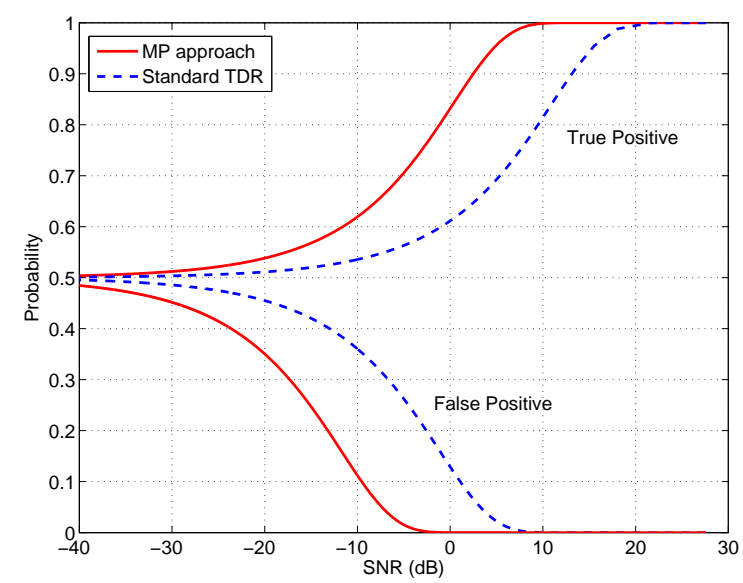

Fig. 12. Detection probabilities and false alarm rates as defined in Section III-B, for the NUT in Fig. 2(a). The SNR shown on the $\mathrm{x}$-axis is that observed at the output of the Gaussian-pulse TDR. The gain provided by the MP approach, and thus the SNR at the output of the MP approach, were estimated from the experimental results, as detailed in Section IV. fault can be more reliably detected.

The formulas (29) and (30) introduced in the previous Section allow studying how the increase in the output SNR leads to a higher detection probability, once the detection gain is computed from the experimental results. It is important to notice that these tools (i.e., for the computation of the detection probabilities) are only based on one assumption, that of a Gaussian noise.

The results thus computed are presented in Fig. 12, having set the decision threshold at $80 \%$ of the fault-related peak. These results illustrate the non-negligible improvement of the MP approach on the likelihood of correctly detecting the presence of an eventual fault, since it increases the detection probability while reducing the false-alarm rate without demanding any increase in the input energy.

We repeated the same analysis for the second configuration in Fig. 3, i.e., with a double-Y structure. The output signals obtained with the Gaussian-pulse TDR technique and the MP one are represented in Fig. 13(a). The MP fault-related peak is now about 4.4 times higher than the one obtained with the standard TDR. The contrast between the echo with the highest amplitude and the second maximum is again better in the MP case, with a margin of 1.65 to be compared to 1.62 for the Gaussian pulse excitation. However, in the TDR case, the fault-related echo is again weaker than the subsequent ones, suggesting a source of ambiguity regarding the localization of the fault, as already pointed out in Section III-A. The detection gain, estimated from the first eleven echoes, is $G^{\prime} \geq 5.6 \mathrm{~dB}$. The output signals obtained with a noise source are shown in Fig. 13(b): as the NUT complexity has increased, the MP provides even clearer results, with an easily detectable echo well above the noisy background.

The conditional detection probability and the false-alarm rate are shown in Fig. 14. The corresponding gain in the SNR and the detection probability imply a more reliable detection of the fault. It is noteworthy how standard TDR techniques would have a hard time in this second example. First of all, the localization of the fault would be put in jeopardy by the fact that the strongest echoes are not those closer to the faultrelated one. Moreover, the very weak response from the fault implies that in certain configurations the output signal from a standard TDR technique could be so low as to be close to other spurious signals. In this case, not only the localization but also the detection of the fault can become critical. On the other hand, the MP approach has clearly shown that at least the detection can be improved, by using suitably defined testing signals.

Clearly, when the SNR is high enough, any detection strategy will ultimately work, as the dynamical range of detection will be infinite, i.e., even the slightest modification could be resolved. In practice, one of the criteria for discriminating the performance of the large number of available techniques is the residual margin left for a reliable detection. It is in this respect that the MP can bring a substantial improvement, by increasing the margin on the effective SNR.

These results completely support our conclusions, as derived in Section III. Although the models we applied were quite simple, they are capable of reproducing the main physical 


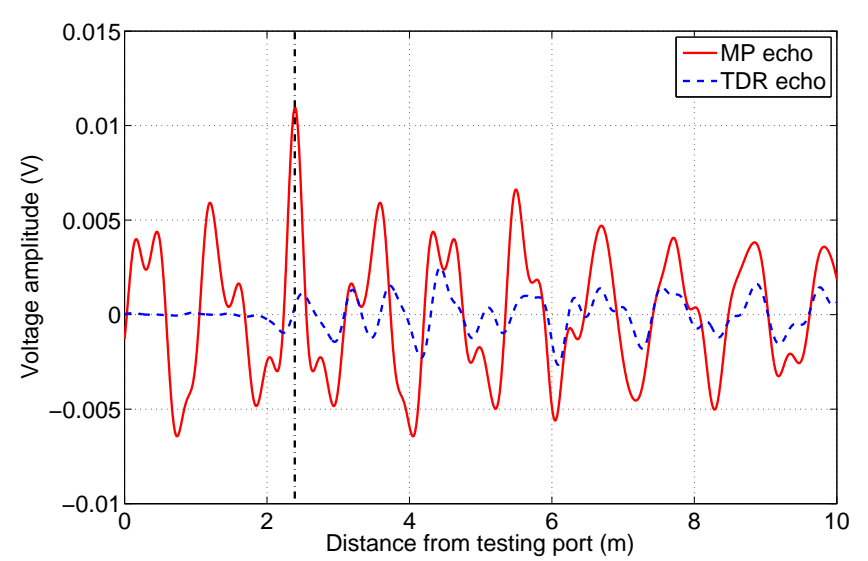

(a)

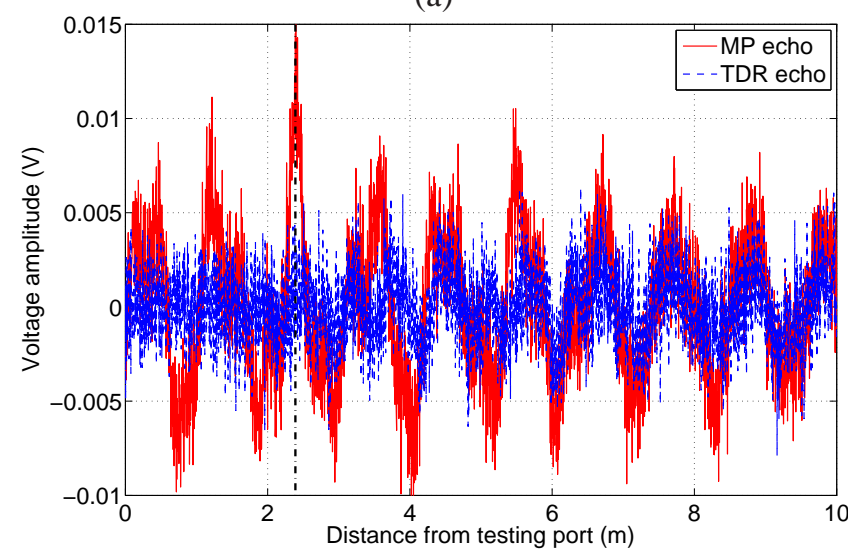

(b)

Fig. 13. The output signals obtained when exciting the network of Fig. 2(b) with open-circuited ends by means of a Gaussian pulse or the associated MP for: (a) a noiseless configuration and (b) the presence of an additive Gaussian noise signal with a fixed average energy, resulting in an SNR of $0 \mathrm{~dB}$ for the Gaussian TDR case, corresponding to an SNR of $12.85 \mathrm{~dB}$ for the MP one. The vertical dashed line represents the position of the fault-related echo.

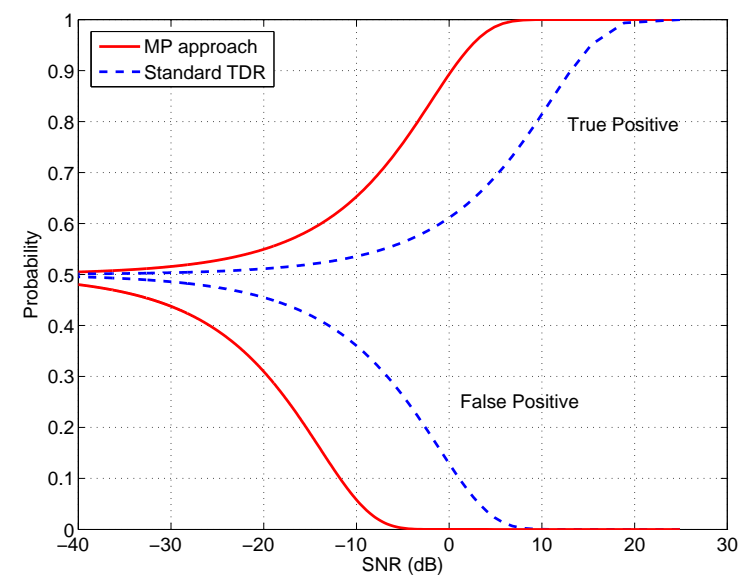

Fig. 14. Detection probabilities and false alarm rates as defined in Section III-B, for the NUT in Fig. 2(b). The SNR shown on the x-axis is that observed at the output of the Gaussian-pulse TDR. The gain provided by the MP approach, and thus the SNR at the output of the MP approach, were estimated from the experimental results, as detailed in Section IV. phenomena underlying the use of MP reflectometry rather than standard ones and predict the trend we have experimentally observed in these results. The most important result is that indeed the use of the MP approach is justified only when dealing with NUTs with a complex response, in order to take advantage of the increased SNR. Moreover, this gain is clearly useful only when the output SNR of a standard TDR approach is deemed too low to ensure a reliable detection of faults. Therefore, the MP approach is indeed confirmed to be an interesting alternative in critical configurations where standard TDR techniques would be at the limit of their capabilities.

It is important to notice that the use of the MP approach can be easily integrated into standard TDR approaches: in the case of an output TDR signal too badly corrupted by noise, this signal would just need to be time-reversed and injected back into the NUT in order to switch to an MP approach. This procedure should allow to improve the reliability of the detection, by simply requiring a further step in the usual TDR sequence shown in Fig. 1.

\section{Conclusions}

An alternative approach for soft-fault detection in complex wire networks was introduced, based on the properties of matched-filter signal processing. The proposed method is based on a self-adaptive definition of the testing signal, which has the peculiarity of ensuring the highest fault-related echo with respect to a given injected energy.

A mathematical analysis based on a simple yet general propagation model and experimental validations have proven that the MP approach outperforms standard TDR methods, as long as the detection probability is of concern. Moreover, it was shown that the complexity of the response of the NUT has a positive effect on the performance of the MP method, as an increasingly longer sequence of echoes systematically leads to a higher fraction of energy being concentrated into the peak echo. This remarkable feature is opposed to common understanding usually based on the properties of standard TDR techniques, where complex-network responses can strongly reduce the reliability of the detection criteria currently used. In a similar way, the MP approach was also shown to benefit from dispersive responses of the fault, as its matched-filter nature allows adapting the testing pulse to the frequency-spectrum of the fault.

As a consequence, the MP approach can be expected to provide a complementary testing solution with major advantages in critical configurations where standard TDR techniques are no more reliable, by ensuring a higher sensitivity to the presence of soft faults.

\section{REFERENCES}

[1] C. Furse and R. Haupt, "Down to the wire," IEEE Spectrum, vol. 38, no. 2, pp. 34-39, 2001.

[2] "Review of federal programs for wire system safety," National Science and Technology Council, White House, Final Report, November 2000.

[3] C. Teal and C. Satterlee, "Managed aircraft wiring health directly relates to improved avionics performance," in Digital Avionics Systems Conference, Philadelphia, 2000.

[4] P. Tsai, C. Lo, Y. C. Chung, and C. Furse, "Mixed-signal reflectometer for location of faults on aging wiring," Sensors Journal, IEEE, vol. 5, no. 6, pp. 1479-1482, December 2005. 
[5] V. Telasula, C. Furse, and C. Lo, "Selection criteria of test signals for correlation-based wire fault analysis," Air Force Research Laboratory, Tech. Rep., 2006.

[6] Y.-J. Shin, E. Powers, T.-S. Choe, C.-Y. Hong, E.-S. Song, J.-G. Yook, and J. B. Park, "Application of time-frequency domain reflectometry for detection and localization of a fault on a coaxial cable," Instrumentation and Measurement, IEEE Transactions on, vol. 54, no. 6, pp. 2493 2500, December 2005.

[7] T.-W. Pan, C.-W. Hsue, and J.-F. Huang, "Time-domain reflectometry using arbitrary incident waveforms," Microwave Theory and Techniques, IEEE Transactions on, vol. 50, no. 11, pp. 2558 - 2563, November 2002.

[8] C. Furse, Y. C. Chung, R. Dangol, M. N. G. Mabey, and R. Woodward, "Frequency-domain reflectometery for on-board testing of aging aircraft wiring," IEEE Transactions on Electromagnetic Compatibility, vol. 45, no. 2, pp. 306-315, May 2003.

[9] P. Smith, C. Furse, and J. Gunther, "Analysis of spread spectrum time domain reflectometry for wire fault location," IEEE Sensors Journal, vol. 5, no. 6, pp. 1469-1478, December 2005.

[10] C. Lo and C. Furse, "Noise-domain reflectometry for locating wiring faults," IEEE Transactions on Electromagnetic Compatibility, vol. 47, no. 1, pp. 97-104, February 2005.

[11] S. Naik, C. Furse, and B. Farhang-Boroujeny, "Multicarrier reflectometry," Sensors Journal, IEEE, vol. 6, no. 3, pp. 812 -818, June 2006.

[12] L. Griffiths, R. Parakh, C. Furse, and B. Baker, "The invisible fray: A critical analysis of the use of reflectometry for fray location," Sensors Journal, IEEE, vol. 6, no. 3, pp. 697-706, June 2006.

[13] C.-W. Hsue and T.-W. Pan, "Reconstruction of nonuniform transmission lines from time-domain reflectometry," Microwave Theory and Techniques, IEEE Transactions on, vol. 45, no. 1, pp. 32 -38, January 1997.

[14] A. Derode, A. Tourin, J. de Rosny, M. Tanter, S. Yon, and M. Fink, "Taking advantage of multiple scattering to communicate with timereversal antennas," Physical Review Letters, vol. 90, no. 1, p. 14301, 2003.

[15] A. Derode, P. Roux, and M. Fink, "Robust acoustic time reversal with high-order multiple scattering," Physical Review Letters, vol. 75, no. 23, pp. 4206-4209, 1995.

[16] A. Derode, A. Tourin, and M. Fink, "Random multiple scattering of ultrasound. II. Is time reversal a self-averaging process?" Physical Review E, vol. 64, no. 3, p. 36606, 2001.

[17] J.-G. Zhu, Y. Jiang, D. Stancil, and J. Moura, "A novel time reversal method for target detection in cluttered media," vol. 4B, July 2005, pp. 135 - 138 vol. 4B.

[18] Y. Jiang, D. Stancil, and J.-G. Zhu, "Antenna array detection in highly cluttered environment using time reversal method," in Microwave Symposium, 2007. IEEE/MTT-S International, Honolulu, June 2007, pp. 1731-1734.

[19] J. M. F. Moura and Y. Jin, "Detection by time reversal: Single antenna," Signal Processing, IEEE Transactions on, vol. 55, no. 1, pp. 187 -201, January 2007.

[20] M. Fink, "Time reversal of ultrasonic fields - part 1: Basic principles," IEEE Transactions on Ultrasonics, Ferroelectrics, and Frequency Control, vol. 39, no. 5, pp. 555-566, September 1992.

[21] L. Abboud, A. Cozza, and L. Pichon, "Utilization of matched pulses to improve fault detection in wire networks." Lille: 9th International Conference on ITS Telecommunications, October 2009.

[22] _ , "Performance analysis of the matched-pulse-based fault detection," in Proceedings of the First International Conference on Complex Systems Design and Management, D. Krob, Ed., Paris, October 2010, pp. 161-172.

[23] G. Cerri, R. De Leo, L. Della Nebbia, S. Pennesi, V. Primiani, and P. Russo, "Fault locatio o shielded cables: Electromagnetic modelling and improved measurement data processing," in Science, Measurement and Technology, IEE Proceedings-, vol. 152, no. 5. IET, 2005, pp. 217-226.

[24] P. Kahn, Mathematical Methods for Scientists and Engineers: Linear and Nonlinear Systems. New York: J. Wiley, 1990.

[25] D. Ricker, Echo signal processing. Springer Netherlands, 2003, vol. 725.

[26] P. Dunn, Measurement and Data Analysis for Engineering and Science. CRC Press Taylor and Francis group, 2010.

[27] C. Buccella, M. Feliziani, and G. Manzi, "Detection and localization of defects in shielded cables by time-domain measurements with UWB pulse injection and clean algorithm postprocessing," Electromagnetic Compatibility, IEEE Transactions on, vol. 46, no. 4, pp. 597 - 605, November 2004. 